\title{
Chitosan and Chitosan Derivatives for Biological Applications: Chemistry and Functionalization
}

\author{
Bruno Sarmento, ${ }^{1,2}$ Francisco M. Goycoolea, ${ }^{3}$ Alejandro Sosnik,,${ }^{4,5}$ and José das Neves ${ }^{1}$ \\ ${ }^{1}$ Department of Pharmaceutical Technology, Faculty of Pharmacy, University of Porto, Rua Aníbal Cunha 164, 4050-047 Porto, Portugal \\ ${ }^{2}$ CICS, Department of Pharmaceutical Sciences, Instituto Superior de Ciências da Saúde-Norte, Gandra, Portugal \\ ${ }^{3}$ Institut für Biologie und Biotechnologie der Pflanzen, Westfälische Wilhelms Universtät-Münster, Hindenburgplatz 55, \\ 48143 Münster, Germany \\ ${ }^{4}$ The Group of Biomaterials and Nanotechnology for Improved Medicines (BIONIMED), Department of Pharmaceutical Technology, \\ Faculty of Pharmacy and Biochemistry, University of Buenos Aires, 6th Floor, 956 Junin Street, CP 1113 Buenos Aires, Argentina \\ ${ }^{5}$ National Science Research Council (CONICET), Buenos Aires, Argentina \\ Correspondence should be addressed to Bruno Sarmento, bruno.sarmento@ff.up.pt
}

Received 12 October 2011; Accepted 12 October 2011

Copyright ( $) 2011$ Bruno Sarmento et al. This is an open access article distributed under the Creative Commons Attribution License, which permits unrestricted use, distribution, and reproduction in any medium, provided the original work is properly cited.

It is with great satisfaction that we present this special issue in the International Journal of Carbohydrate Chemistry. Chitosan chemical derivatives have received increasing interest over the past decades due to their advocated chemical, biological, and functional advantages over unmodified chitosan. These include, but are not limited to, better solubility in aqueous solutions over a wider range of $\mathrm{pH}$, gelling properties, possibility to revert the net charge from polycationic to polyanionic, design of hydrophobic derivatives with amphiphilic character and capacity to harness selfassembling nanostructures and chemical conjugates with an assortment of bioactive and therapeutic molecules; improved biocompatibility (e.g., hemocompatibility); enhanced properties for complexing pDNA or siRNA.

Undoubtedly, life sciences and technologies is the realm where chitosan and chitosan derivatives have raised greater scientific interest. A recent search in the Web of Knowledge ${ }^{\mathrm{SM}}$ reveals that more than half out of the total number of published papers and patents including the terms "chitosan" or "chitosan derivatives" $(>54000)$ published over the past two decades are directly related to the life sciences. The number of papers published in this field has kept a sustained increasing trend over the past years, and only in 2010 a total of ca. 1800 papers have appeared in this field. This analysis clearly illustrates that the interest in chitosan in the biological sciences continues to increase and to receive funding by research agencies worldwide.
In this special issue of the International Journal of Carbohydrate Chemistry, we have put together a collection of six papers (five reviews and one research article). The review papers provide an overview of the state of the art of a large repertoire of chitosan derivatives and materials derived from them in various fields of life sciences, spanning pulmonary drug delivery (F. Andrade et al.), DNA condensation (K. E. Kador and A. Subramanian), biomaterials for tissue regeneration (G. D. Guerra et al.), food microbiology (D. de Britto et al.), and crop protection (M. E. I. Badawy and E. I. Rabea). In turn, the research article (S. E. Noriega and A. Subramanian) focuses on chitosan films for chondrocytes culture. The areas encompassed by these papers are among the ones that currently represent the most prominent opportunities for the utilization of chitosan and chitosan derivatives; hence, it is foreseeable that the interest in the recent advances in the field will continue but to increase in the coming years.

We are confident that this special issue will attract the attention of a wide audience worldwide that will also benefit from the many advantages associated to open access journals like the International Journal of Carbohydrate Chemistry.

\footnotetext{
Bruno Sarmento Francisco Goycoolea Alejandro Sosnik José das Neves
} 


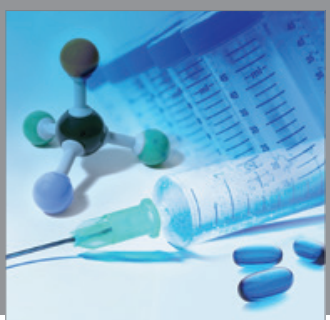

International Journal of

Medicinal Chemistry

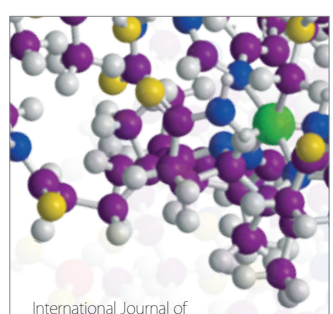

Carbohydrate Chemistry

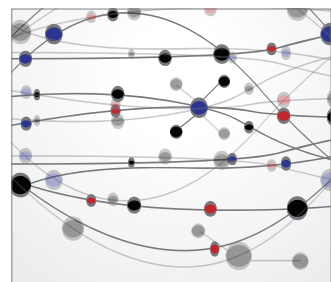

The Scientific World Journal
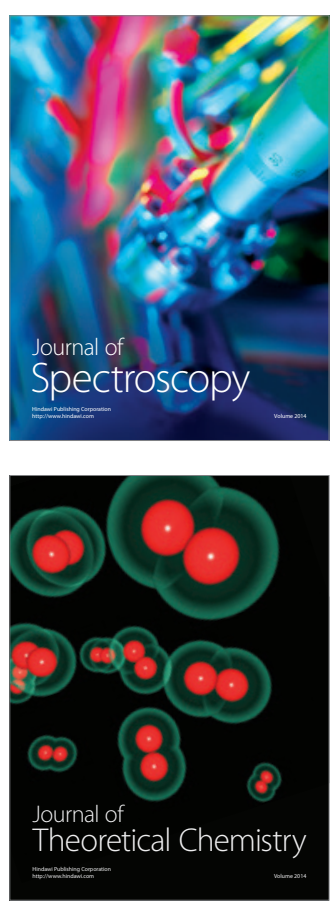
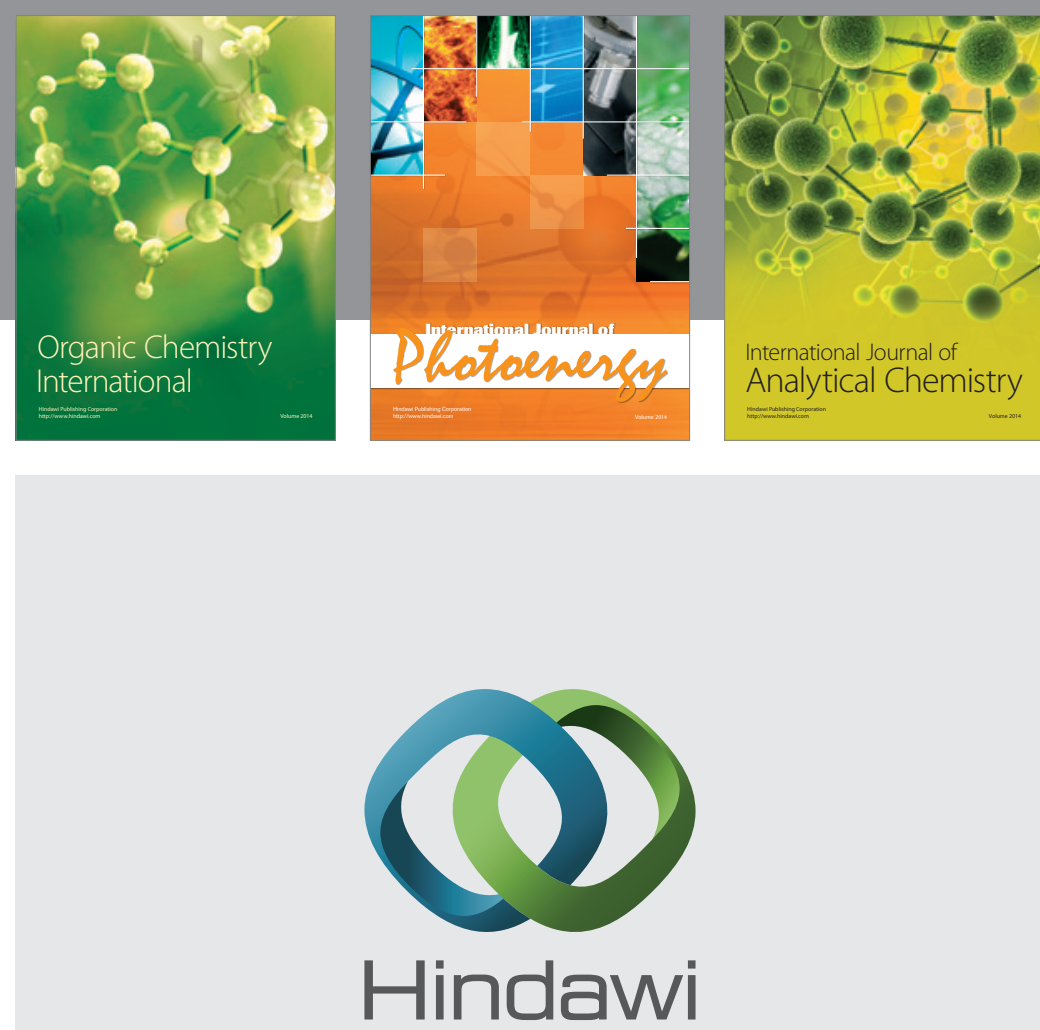

Submit your manuscripts at

http://www.hindawi.com
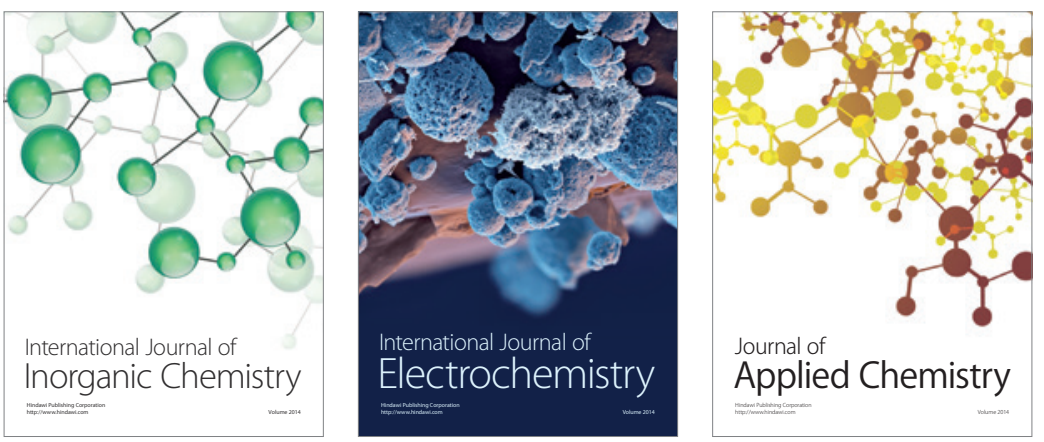

Journal of

Applied Chemistry
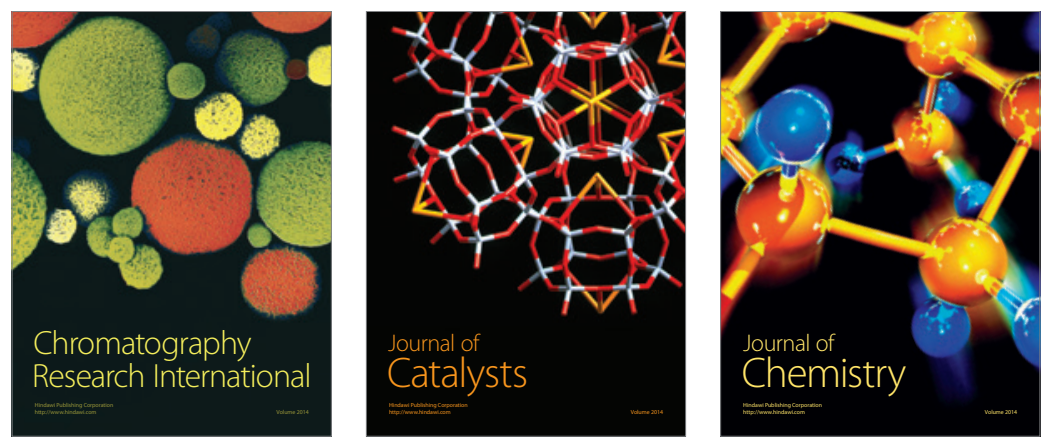
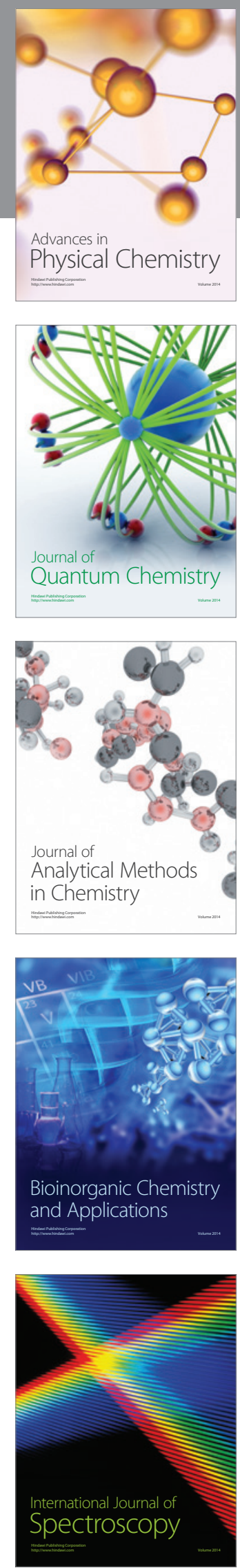